\title{
Monoacylglycerol lipase inhibitor JZL184 reduces neuroinflammatory response in APdE9 mice and in adult mouse glial cells
}

Rea Pihlaja ${ }^{1 *}$, Jatta Takkinen ${ }^{1}$, Olli Eskola², Jenni Vasara ${ }^{1}$, Francisco R López-Picón ${ }^{1}$, Merja Haaparanta-Solin ${ }^{1}$ and Juha O Rinne ${ }^{3}$

\begin{abstract}
Background: Recently, the role of monoacylglycerol lipase (MAGL) as the principal regulator of simultaneous prostaglandin synthesis and endocannabinoid receptor activation in the CNS was demonstrated. To expand upon previously published research in the field, we observed the effect of the MAGL inhibitor JZL184 during the early-stage proinflammatory response and formation of beta-amyloid (A $\beta$ ) in the Alzheimer's disease mouse model APdE9. We also investigated its effects in proinflammatory agent - induced astrocytes and microglia isolated from adult mice.

Findings: Transgenic APdE9 mice (5 months old) were treated with JZL184 (40 mg/kg) or vehicle every day for 1 month. In vivo binding of the neuroinflammation-related, microglia-specific translocator protein (TSPO) targeting radioligand $\left[{ }^{18} \mathrm{~F}\right] \mathrm{GE}-180$ decreased slightly but statistically non-significantly in multiple brain areas compared to vehicle-treated mice. JZL184 treatment induced a significant decrease in expression levels of inflammation-induced, Iba1-immunoreactive microglia in the hippocampus $(P<0.01)$ and temporal and parietal $(P<0.05)$ cortices. JZL184 also induced a marked decrease in total $A \beta$ burden in the temporal $(P<0.001)$ and parietal $(P<0.01)$ cortices and, to some extent, in the hippocampus. Adult microglial and astrocyte cultures pre-treated with JZL184 and then exposed to the neuroinflammation-inducing agents lipopolysaccharide (LPS), interferon-gamma (IFN- $\gamma$ ), and $A \beta_{42}$ had significantly reduced proinflammatory responses compared to cells without JZL184 treatment.
\end{abstract}

Conclusions: JZL184 decreased the proinflammatory reactions of microglia and reduced the total A $\beta$ burden and its precursors in the APdE9 mouse model. It also reduced the proinflammatory responses of microglia and astrocytes isolated from adult mice.

Keywords: Alzheimer's disease, Neuroinflammation, Monoacylglycerol lipase

\section{Findings}

During early-stage Alzheimer's disease (AD), the neuroinflammatory responses of hyperactivated glial cells have a major impact on the development of $\mathrm{AD}$ pathology, including the formation of neurotoxic betaamyloid $(A \beta)[1,2]$. Suppressing inflammatory signaling has had beneficial effects in animal models of AD [3]. On the other hand, molecular imaging of neuroinflammation may be employed for the early diagnosis of $\mathrm{AD}$ and to evaluate the patient's response to treatment.

\footnotetext{
* Correspondence: rea.pihlaja@utu.fi

${ }^{1}$ MediCity/PET Preclinical Laboratory, Turku PET Centre, University of Turku, Tykistökatu 6 A, Fl-20520 Turku, Finland

Full list of author information is available at the end of the article
}

In the brain, the production of proinflammatory prostaglandins is mainly regulated by the activity of monoacylglycerol lipase (MAGL) [4]. MAGL hydrolyzes the anti-inflammatory and neuroprotective endocannabinoid 2-arachidonoylglycerol (2-AG) to arachidonic acid (AA), which is further metabolized to prostaglandins. Inhibition of MAGL by genetic manipulation or the MAGL inhibitor JZL184 reduces the levels of AA and its prostaglandin metabolites E2 and D2. Inhibition of MAGL also increases levels of 2-AG in the brain. Both of these mechanisms are associated with neuroprotection in a parkinsonian mouse model and alleviation of lipopolysaccharide (LPS)-induced neuroinflammation. Moreover, pharmacological [5] and genetic [6] inactivation of MAGL 
markedly suppresses $A \beta$ load, reduces neuropathology, and improves cognitive function in the $\mathrm{AD}$ mouse models 5XFAD and PS1APP. To date, of the MAGL inhibitors, JZL184 has been the most thoroughly characterized in vivo; thus, this compound is widely used as a preclinical tool [7].

We extended the findings of previous studies by investigating the effect of JZL184 on the early-stage proinflammatory response and $A \beta$ deposition in APPSwe/ PSEN1dE9 (APdE9) [8] mice. The level of activated microglia in JZL184- and vehicle-treated mice was determined by positron emission tomography-computed tomography (PET/CT). Levels of gliosis and total $\mathrm{A} \beta$ were determined by immunohistochemistry. In addition, we studied the effect of JZL184 in glial cultures isolated from adult mice under conditions that mimic neuroinflammation.

In 3.5-month-old APdE9 mice, levels of proinflammatory mediators are correlated with soluble $A \beta$ levels. By the age of 6 months, the proinflammatory responses of glial cells notably increased and the first $A \beta$ deposits have formed [2]. For in vivo studies, we used 5-monthold female APdE9 mice. The studies were approved by the Animal Experiment Board of the Province of Southern Finland (licence number ESAVI-2010-04454/Ym-23). JZL184 (Cayman Chemical Company, Ann Arbor, MI, USA) formulation was prepared in an 18:1:1 solution of saline:emulphor:ethanol, as previously described [7]. Every day for 1 month, $40 \mathrm{mg} / \mathrm{kg} \mathrm{JZL184}$ was injected intraperitoneally into $\operatorname{tg}$ APdE9 mice $(n=7)$. Vehicle-treated tg APdE9 mice $(n=5)$ were used as controls.

Mice were anesthetized with $2.5 \%$ isoflurane, and $15 \pm$ $2 \mathrm{MBq}$ of radioligand $\left[{ }^{18} \mathrm{~F}\right] \mathrm{GE}-180$ [9] was injected intravenously. A 60-min dynamic PET/CT scan (Inveon multimodality PET/CT device; Siemens Medical Solutions, Malvern, PA, USA) was started immediately after injection (3D list mode; energy window of 350 to $650 \mathrm{keV}$; and 52 time frames: $30 \times 10,15 \times 60,4 \times 300$, and $2 \times 600 \mathrm{~s})$. Transmission scans were performed with CT for attenuation correction. Data were reconstructed with Fourier rebinning and a 2D-filtered back-projection reconstruction algorithm. Inveon Research Workplace Image Analysis software (Siemens Medical Solutions) was used to draw regions of interest (ROIs) over the whole brain, the cerebellum, and the entire cortex and separately over the frontal and parieto-temporal cortex, hippocampus, striatum, and thalamus. A 3D MRI template was used as an anatomical reference. Time-radioactivity curves were used to express the uptake of $\left[{ }^{18} \mathrm{~F}\right] \mathrm{GE}-180$ as $\% \mathrm{ID} / \mathrm{g}$, and target-to-cerebellum ratios were calculated. In JZL184-treated mice, there was a very slight decreasing trend in translocator protein (TSPO) signal in multiple ROIs (for example, in the hippocampus, Figure 1B) compared to vehicle-treated mice. However, the difference was not statistically significant.
All JZL184 $(n=7)$ and vehicle $(n=5)$-treated mice were anesthetized and transcardially perfused with heparinized saline. The cortex, hippocampus, and cerebellum were then isolated from the right hemisphere and snap frozen in liquid nitrogen. The left hemisphere was postfixed with $4 \%$ paraformaldehyde solution, cryoprotected in $30 \%$ sucrose, snap frozen in liquid nitrogen, and stored at $-70^{\circ} \mathrm{C}$. Processing of the brain tissue and immunohistochemistry were performed as described previously [10], using the mouse beta-amyloid monoclonal antibody 6E10 (Covance Inc., Princeton, NJ, USA) and a polyclonal rabbit anti-glial fibrillary acidic protein (GFAP) antibody (Dako Cytomation, Glostrup, Denmark) that recognizes activated astrocytes or a rabbit polyclonal antiIba1 antibody (WAKO Pure Chemical Industries, Ltd., Osaka, Japan). The 6E10 antibody recognizes amino acid residues 1-16 in various abnormally processed isoforms of intra- and extracellular human $\mathrm{A} \beta$ [11-15], as well as amyloid precursors. Alexa Fluor 568-conjugated goat antirabbit (Gibco, Carlsbad, CA, USA) or donkey anti-mouse IgG (Dylight 488, Abcam, Cambridge, UK) were used as secondary antibodies. Images were acquired with the Leica DMR fluorescence microscope (Leica Microsystems $\mathrm{GmbH}$, Wetzlar, Germany) and ISCapture software 2.6 (Fuzhou Xintu Photonics Co, Ltd., Fujian, China); settings were kept constant for quantitation. Six or seven sections per mouse with five to seven areas per section were imaged throughout the hippocampal formation. The areas examined were the temporal and parietal cortices and the hippocampus (including CA1, CA2, and DG). The quantification was performed using per area (\%) method (Image J 1.43 U, Wayne Rasband, NIH, MA, USA).

Levels of Iba1 expression were decreased in JZL184treated mice in the temporal and parietal cortices (by $49 \%$ and $46 \%$, respectively; $P<0.05)$ and in the hippocampus (by $57 \%, P<0.01$ ) compared to vehicle-treated mice (Figures 1A and 2A, E, I, M). Similarly, expression levels of GFAP decreased slightly but not significantly in all of the regions examined (Figure 1C). Levels of 6 E10 immunoreactivity were reduced significantly in the temporal (by 79\%, $P<0.001$ ) and parietal (by $67 \%$, $P<0.01)$ cortices and, to some extent, in the hippocampus compared to vehicle-treated mice (Figures $1 \mathrm{D}$ and 2B, F, J, N).

A human $A \beta_{42}$ enzyme-linked immunosorbent assay (ELISA) was performed for the hippocampi and cortices isolated from the APdE9 mice, in accordance with the protocol for the A $\beta 42$ Human ELISA Kit (Biosource International/Invitrogen, Carlsbad, CA, USA). Expression levels of $A \beta_{42}$ in the hippocampus and cortex in ELISA did not differ between JZL184- and vehicletreated tg APdE9 mice (data not shown). In contrast to $6 \mathrm{E} 10$ antibody, $A \beta_{42}$ antibody in ELISA recognizes only C-terminus of the 1-42 A $\beta$ sequence. 

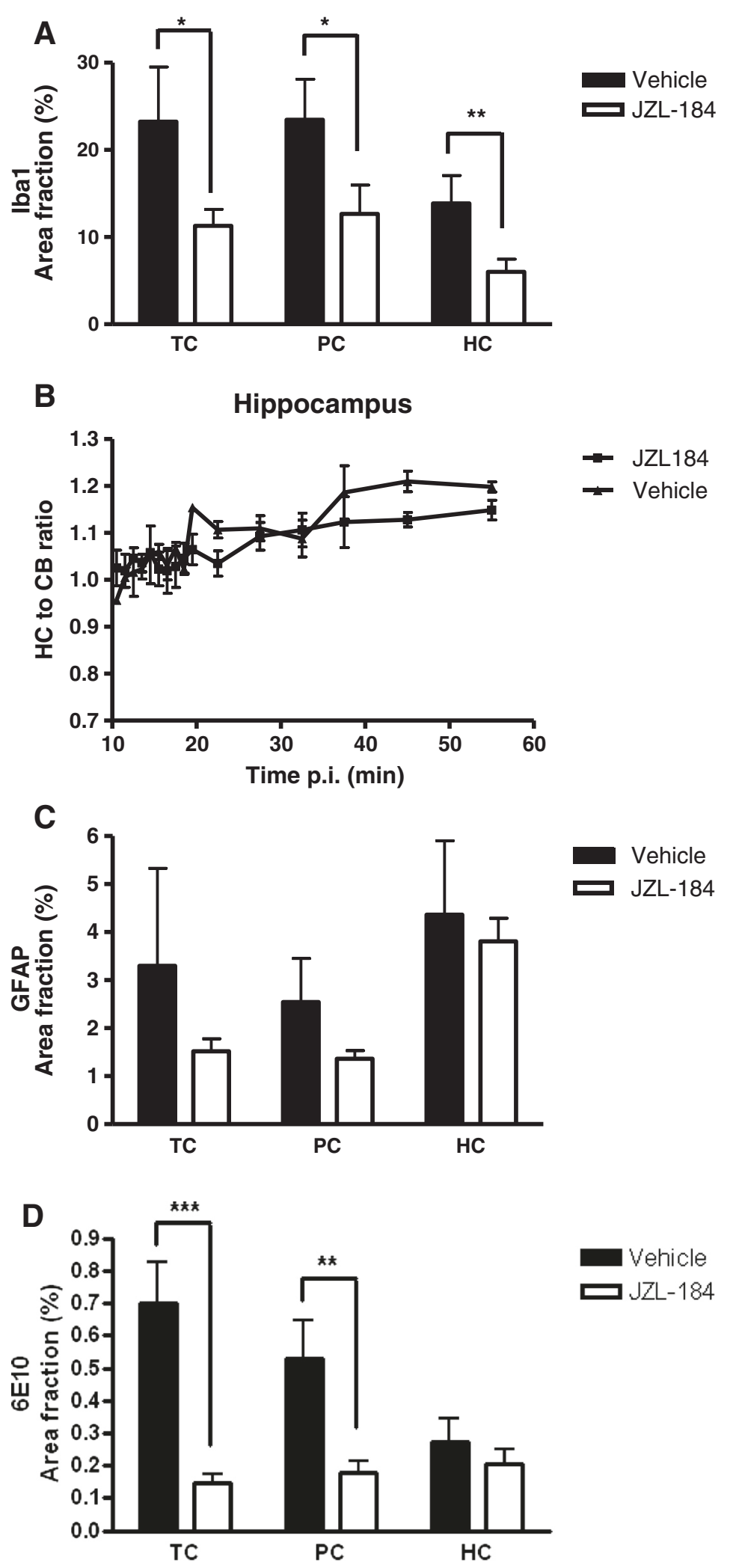

Figure 1 (See legend on next page.) 
(See figure on previous page.)

Figure 1 JZL184 treatment reduced the level of microgliosis and total A $\beta$ in the AD mouse model. One month daily treatment with JZL184 (40 mg/kg, i.p.) reduced the immunoreactivity of $(\mathbf{A}) \mathrm{lba} 1$ in temporal $(\mathrm{TC})(P<0.05)$ and parietal $(P C)(P<0.05)$ cortices and hippocampus $(\mathrm{HC})(P<0.01)$ in 5-month-old tg APdE9 mice when compared to vehicle-treated mice. Also, (B) TSPO targeting radioligand [ ${ }^{18}$ F]GE-180 decreased slightly but statistically non-significantly in multiple brain areas, for example, in the hippocampi. The level of (C) GFAP did not decrease significantly. The level of (D) 6 E10 was reduced in TC $(P<0.001)$ and $P C(P<0.01)$ and slightly in $H C$.

For the cell culture experiments, adult microglia and astrocytes were cultured according to the procedures outlined in [16] and [17], respectively. Three days before exposure, the cells were seeded $\left(2 \times 10^{4}\right.$ cells/well $)$ into 48-well plates in the absence of recombinant murine granulocyte-macrophage colony-stimulating factor (rmGM-CSF) and G5 supplement.

Homogenous $A \beta_{42}$ (American Peptide Company, Sunnyvale, CA, USA) oligomers were prepared according to protocol by Dahlgren et al. [18]. For cell culture assays, the wells containing adult microglia and astrocytes were rinsed with medium w/o serum, incubated for $30 \mathrm{~min}$ in the presence or absence of $1 \mu \mathrm{M}$ JZL184, and then exposed to $10 \mu \mathrm{M} \mathrm{A} \beta_{42}$ or a combination of $1 \mu \mathrm{M}$ LPS and $100 \mathrm{ng} / \mathrm{ml}$ interferongamma (IFN- $\gamma$ ) for $24 \mathrm{~h}$. The medium was collected for nitric oxide (NO) measurement, and the cells were fixed with $3.7 \%$ formaldehyde, rinsed, and stored in phosphate-buffered saline (PBS).

The concentration of NO in the cell medium was measured by Griess reaction according to the manufacturer's instructions (Sigma-Aldrich). Fifty microliters of Griess reagent was then added to each well. The plate was shaken for $10 \mathrm{~min}$, and the absorbance was measured at $560 \mathrm{~nm}$

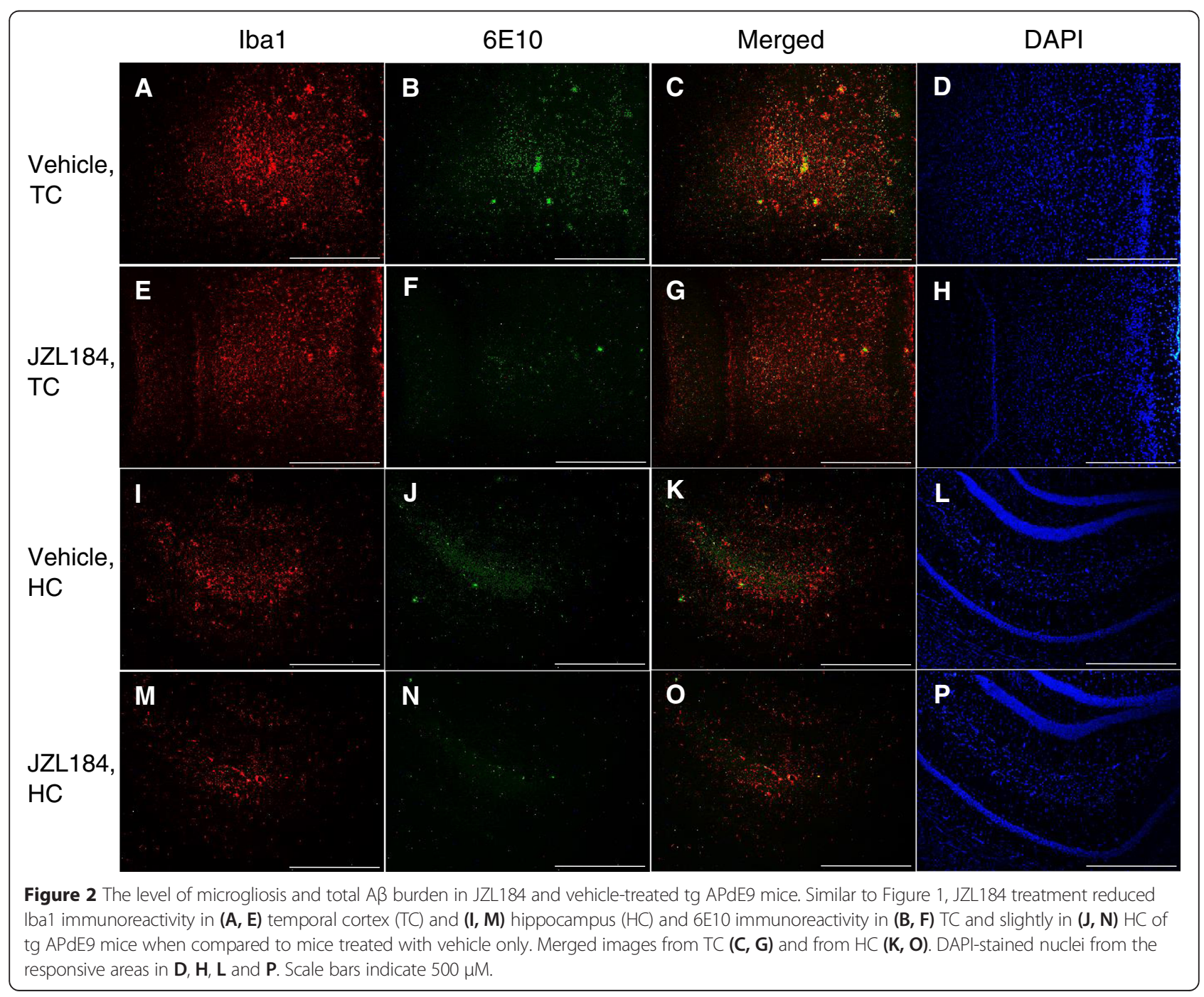


(Wallac Victor2 V 1420; Wallac Oy, Finland). Levels of secreted IL-1 $\beta$ were determined by ELISA according to the manufacturer's instructions (Biosource International/Invitrogen). Simultaneous exposure to LPS and IFN- $\gamma$ was associated with a marked increase in the secretion of $\mathrm{NO}$ and IL-1 $\beta$ in both microglia and astrocytes, compared to unexposed cells (Figure 3A, B, C, D). A $30 \mathrm{~min}$ pretreatment with JZL184 decreased NO secretion slightly in microglia (Figure 3A) and significantly in astrocytes $(P<$ 0.01, Figure 3B) compared to cells w/o JZL184 treatment.
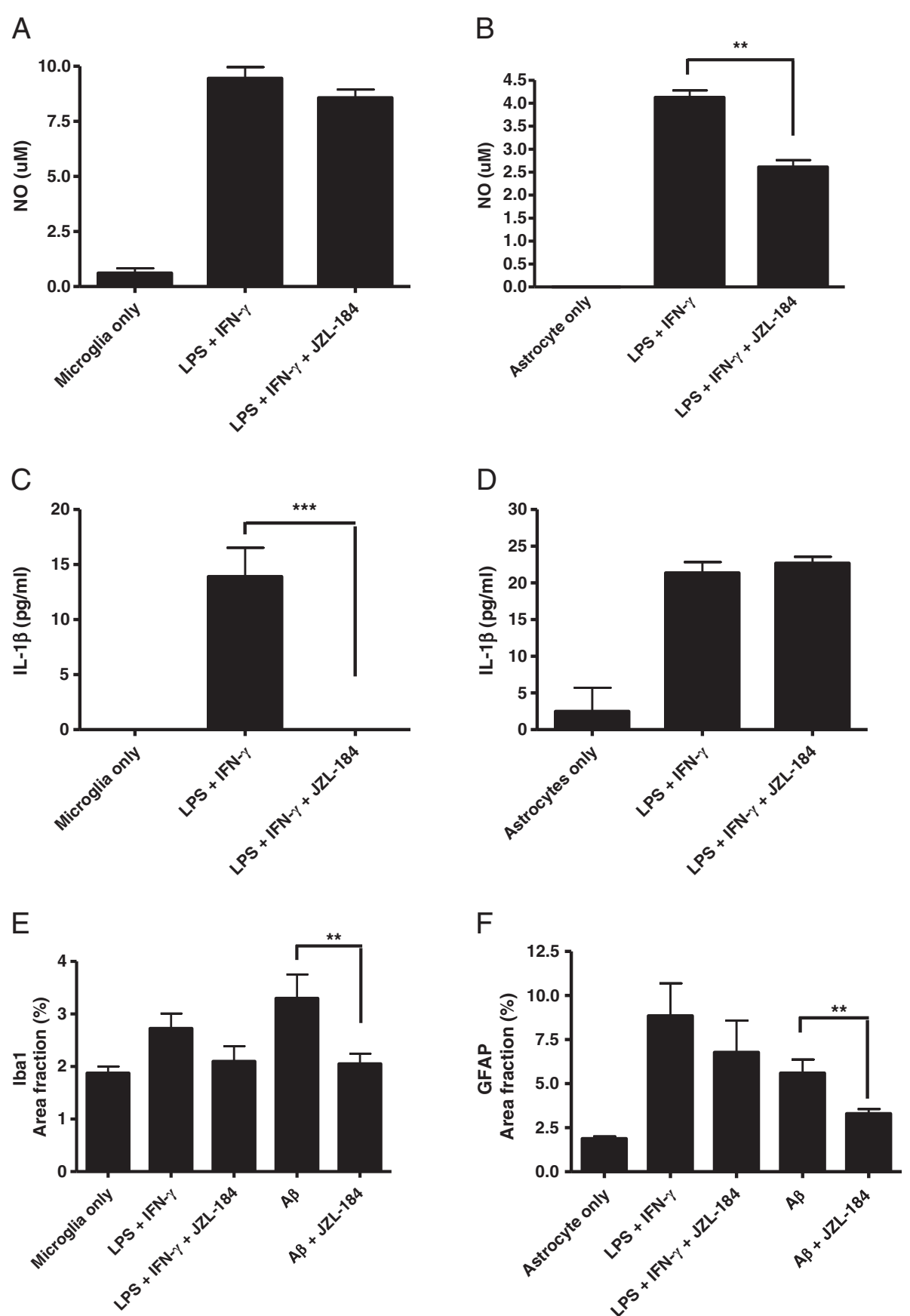

Figure 3 JZL184 decreased secretion of $\mathrm{NO}$ and IL-1 1 from adult glial cells exposed to proinflammatory agents. Astrocytes and microglia were pre-incubated with MAGL inhibitor JZL184 for 30 min before inducing proinflammatory responses by simultaneous exposure of $1 \mu$ M LPS and $100 \mathrm{ng} / \mathrm{ml} \mathrm{IFN-} \gamma$. After $24 \mathrm{~h}$ of incubation, secretion of $\mathrm{NO}$ was reduced $(\mathbf{A})$ slightly in microglia and (B) significantly $(P<0.01)$ in astrocytes treated with JZL184 compared to exposed cells w/o JZL184 treatment. JZL184 induced (C) a marked reduction in IL-1 3 secretion from microglia ( $P<0.001)$ but (D) did not have an effect on astrocytes compared to cells w/o JZL184 treatment. Pre-treatment with JZL184 decreased (E) Iba1 and (F) GFAP expression slightly in microglia and astrocytes induced with LPS and IFN- $\gamma$ and significantly in microglia and astrocytes induced with $A \beta_{42}(P<0.01)$. 
JZL184 pre-treatment abolished the secretion of IL-1 $\beta$ in microglia $(P<0.001$, Figure $3 C)$ but not in astrocytes (Figure 3D) compared to cells w/o JZL184 treatment.

For immunocytochemistry, fixed cells were blocked with 10\% normal goat serum (NGS) (Chemicon) in PBS with Tween-20 (PBST), incubated overnight with either anti-GFAP (in 5\% NGS in PBST) or anti-Iba1-antibodies, and washed in PBST $3 \times$ for 5 min. The secondary antibodies, Alexa Fluor 568 goat anti-rabbit antibody (Gibco) or donkey anti-mouse IgG (Dylight 488, Abcam), were added to the cells, incubated for $2 \mathrm{~h} \mathrm{RT}$, washed, embedded in mounting medium, and imaged with a Zeiss Axiovert $200 \mathrm{M}$ microscope, a Zeiss Axiovert MRc camera, and Axiovision 4.8 software (Zeiss International, Oberkochen, Germany). Four microscopic fields and four to five wells per cell type were analyzed for the percentage of anti-GFAP or anti-Iba immunostained cells, quantified using per area (\%) method (Image J $1.43 \mathrm{U}$ ).

$\mathrm{A} \beta_{42}$ or simultaneous LPS and IFN- $\gamma$ exposure increased Iba1 and GFAP expression. Pre-treatment with JZL184 decreased Iba1 expression significantly in microglia induced with $\mathrm{A} \beta_{42}(P<0.01)$ and slightly when induced with LPS and IFN- $\gamma$ (Figure 3E). Similarly, JZL184 pre-treatment significantly reduced GFAP expression in astrocytes induced with $\mathrm{A} \beta_{42} \quad(P<0.01)$ and slightly when induced with LPS and IFN- $\gamma$ (Figure 3F).

The data were analyzed with $t$-test, one-way ANOVA with Tukey's multiple comparison test, or Mann-Whitney $U$-test with GraphPad Prism v4.0. Significance was assumed if the $P$ value was $<0.05$.

\begin{abstract}
Abbreviations
2-AG: 2-arachidonoylglycerol; AA: arachidonic acid; AD: Alzheimer's disease; Aß: beta-amyloid; CA: cornu ammonis; CT: computed tomography; DG: dentate gyrus; DMSO: dimethyl sulphoxide; ELISA: enzyme-linked immunosorbent assay; GFAP: glial fibrillary acidic protein; lba1: ionized calcium binding adapter molecule-1; IFN-Y: interferon-gamma; LPS: lipopolysaccharide; MAGL: monoacylglycerol lipase; $\mathrm{NaNO}_{2}$ : sodium nitrite; NGS: normal goat serum; NO: nitric oxide; PBS: phosphate-buffered saline; PBST: phosphate-buffered saline with Tween-20; PET/CT: positron emission tomography-computed tomography; ROI: region of interest; tg: transgenic; TSPO: translocator protein.
\end{abstract}

\section{Competing interests}

The authors declare that they have no competing interests.

\section{Authors' contributions}

RP designed the experiments, performed and analyzed the experiments, coordinated collaborations, and wrote the manuscript. JT analyzed the results concerning PET. JV performed the ELISA measurement. OE, FLP, MHS, and JR coordinated collaborations and designed and analyzed the experiments. All authors checked and approved the final manuscript.

\section{Acknowledgements}

The authors thank Dr. Simon Moussaud (Mayo Clinic, Florida, USA) for methodological support concerning adult microglial cultures. This work was funded by the European Community's Seventh Framework Programme (FP7/ 2007-2013) under grant agreement no. HEALTH-F2-2011-278850 (INMiND), the Jane and Aatos Erkko Foundation of Finland, Turku University Hospital (EVO) and GE Healthcare Ltd., which also provided the cassettes for radiotracer production.

\section{Author details}

'MediCity/PET Preclinical Laboratory, Turku PET Centre, University of Turku, Tykistökatu 6 A, Fl-20520 Turku, Finland. ${ }^{2}$ Turku PET Centre,

Radiopharmaceutical Chemistry Laboratory, University of Turku, Turku, Finland. ${ }^{3}$ Turku PET Centre, Turku University Hospital, University of Turku,

Turku, Finland.

Received: 13 March 2014 Accepted: 22 April 2015

Published online: 28 April 2015

\section{References}

1. Ferretti MT, Bruno MA, Ducatenzeiler A, Klein WL, Cuello AC. Intracellular $A \beta$-oligomers and early inflammation in a model of Alzheimer's disease. Neurobiol Aging. 2012;33:1329-42.

2. Zhang W, Bai M, Xi Y, Hao J, Zhang Z, Su C, et al. Multiple inflammatory pathways are involved in the development and progression of cognitive deficits in APPswe/PS1dE9 mice. Neurobiol Aging. 2012;33:2661-77.

3. Wyss-Coray T, Rogers J. Inflammation in Alzheimer disease - a brief review of the basic science and clinical literature. Cold Spring Harb Perspect Med. 2012;2:a006346.

4. Nomura DK, Morrison BE, Blankman JL, Long JZ, Kinsey SG, Marcondes MC et al. Endocannabinoid hydrolysis generates brain prostaglandins that promote neuroinflammation. Science. 2011;334:809-13.

5. Chen R, Zhang J, Wu Y, Wang D, Feng G, Tang YP, et al. Monoacylglycerol lipase is a therapeutic target for Alzheimer's disease. Cell Rep. 2012;2:1329-39.

6. Piro JR, Benjamin DI, Duerr JM, Pi Y, Gonzales C, Wood KM, et al. A dysregulated endocannabinoid-eicosanoid network supports pathogenesis in a mouse model of Alzheimer's disease. Cell Rep. 2012:1:617-23.

7. Long JZ, Nomura DK, Cravatt BF. Characterization of monoacylglycerol lipase inhibition reveals differences in central and peripheral endocannabinoid metabolism. Chem Biol. 2009;16:744-53.

8. Jankowsky JL, Fadale DJ, Anderson J, Xu GM, Gonzales V, Jenkins NA, et al. Mutant presenilins specifically elevate the levels of the 42 residue beta-amyloid peptide in vivo: evidence for augmentation of a 42-specific gamma secretase. Hum Mol Genet. 2004;13:159-70.

9. Wadsworth $\mathrm{H}$, Jones PA, Chau WF, Durrant C, Fouladi N, Passmore J, et al. $\left[^{18} \mathrm{~F}\right] \mathrm{GE}-180$ : a novel fluorine-18 labelled PET tracer for imaging Translocator protein 18 kDa (TSPO). Bioorg Med Chem Lett. 2012;22:1308-13.

10. Pihlaja R, Koistinaho J, Kauppinen R, Sandholm J, Tanila H, Koistinaho M. Multiple cellular and molecular mechanisms are involved in human $A \beta$ clearance by transplanted adult astrocytes. Glia. 2011;59:1643-57.

11. Dineley KT, Xia X, Bui D, Sweatt JD, Zheng H. Accelerated plaque accumulation, associative learning deficits, and up-regulation of alpha 7 nicotinic receptor protein in transgenic mice co-expressing mutant human presenilin 1 and amyloid precursor proteins. J Biol Chem. 2002;277:22768-80.

12. He P, Zhong Z, Lindholm K, Berning L, Lee W, Lemere C, et al. Deletion of tumor necrosis factor death receptor inhibits amyloid beta generation and prevents learning and memory deficits in Alzheimer's mice. J Cell Biol. 2007;178:829-41.

13. Billings $L M$, Oddo $S$, Green KN, McGaugh JL, LaFerla FM. Intraneuronal $A B$ causes the onset of early Alzheimer's disease-related cognitive deficits in transgenic mice. Neuron. 2005;45:675-88.

14. Lesné S, Koh MT, Kotilinek L, Kayed R, Glabe CG, Yang A, et al. A specific amyloid-beta protein assembly in the brain impairs memory. Nature. 2006:440:352-7.

15. Tampellini D, Magrané J, Takahashi RH, Li F, Lin MT, Almeida CG, et al. Internalized antibodies to the Abeta domain of APP reduce neuronal Abeta and protect against synaptic alterations. J Biol Chem. 2007;282:18895-906.

16. Moussaud S, Draheim HJ. A new method to isolate microglia from adult mice and culture them for an extended period of time. J Neurosci Methods. 2010;187:243-53.

17. Pihlaja R, Koistinaho J, Malm T, Sikkilä H, Vainio S, Koistinaho M. Transplanted astrocytes internalize deposited beta-amyloid peptides in a transgenic mouse model of Alzheimer's disease. Glia. 2008:56:154-63.

18. Dahlgren KN, Manelli AM, Stine Jr WB, Baker LK, Krafft GA, LaDu MJ. Oligomeric and fibrillar species of amyloid-beta peptides differentially affect neuronal viability. J Biol Chem. 2002;277:32046-53. 